\title{
Targeting IL-6 To Calm the Cytokine Release Storm: A Review and Perspective
}

\author{
Shawn J Rice ${ }^{1}$ and Chandra P Belani ${ }^{1,2 *}$ \\ ${ }^{1}$ Penn State Cancer Institute, USA \\ ${ }^{2}$ Department of Medicine, Penn State College of Medicine, Milton S. Hershey Medical Center, USA
}

*Corresponding author: Chandra P Belani, Department of Medicine Penn State College of Medicine, Milton S. Hershey Medical Center, Hershey, PA 17033, USA.

To Cite This Article: El Bouhmadi Khadija, Youssef Oukessou, Sami Rouadi, Redallah Larbi Abada, Mini Review of Malignant Transformation of Benign Parotid Gland Tumors. Am J Biomed Sci \& Res. 2020 - 11(3). AJBSR.MS.ID.001634. DOI: 10.34297/AJBSR.2020.11.001634.

Received: 齿 December 13, 2020; Published: 眥 December 18, 2020

\begin{abstract}
Importance: Cytokine release syndrome (CRS) or cytokine storms can affect many body systems and potentiate life-threatening respiratory distress. Various etiologies can elicit a CRS response such as SARS-CoV-2 infection (Corona Virus Disease 2019; COVID-19), E-cigarette, and Vaping Associated Lung Injury (EVALI), and immune-related adverse events in patients receiving effector cell therapies or immune checkpoint inhibitors. CRS does not occur in all patients thus managing the condition is a challenge. We explore the treatment of CRS across various scenarios to establish a novel approach based on various immunologic parameters and possible activation of cytokines.
\end{abstract}

Observations: Maintaining a robust and functional immune system is essential in cancer patients receiving immunotherapies, i.e., cell-based or immune checkpoint inhibitors (ICI). However, cessation of therapy and muting of the immune system is often required once a patient presents with severe CRS, which can reduce or eliminate any effect of the tumor-fighting agent. Corticosteroids are the most common approach to calming symptoms associated with CRS from all etiologies. More recently, it has been demonstrated that IL-6 is a key component of the CRS response. Thus, it is prudent to combine IL-6 targeted agents with corticosteroids to achieve an optimal effect against CRS.

Conclusions and Relevance: Approaches that utilize IL-6/IL-6R antagonists in combination with steroids could provide adequate efficacy against CRS without affecting the immune-based tumor response. Gaps in knowledge remain for the treatment of patients with CRS, and it continues to provide a dynamic target for optimization of combination strategies.

Keywords: Interleukin; IL-6; Cytokine Release Syndrome; Cytokine Storm

\section{Introduction}

Cytokine Release Syndrome (CRS), also known as Cytokine Storm Syndrome (CSS), is a systemic inflammatory response resulting from an exaggerated and uncontrolled immune response. CRS is associated with life-threatening inflammation that can affect many organs (i.e., pneumonitis, enteritis, colitis, vitiligo, etc.). Uncontrolled CRS could result in multiple organ failure and death. As the name implies, a central characteristic of CRS is elevated systemic cytokine levels due to excessive activation of immune and effector cells. A range of symptoms is associated with CRS from mild symptoms like fever, headache, rash, fatigue, and myalgia, to more severe symptoms like hypotension, high-grade fever, vascular permeability, and disseminated intravascular coagulation1.
Inflammation in the lungs can result in severe cough and elevated respiratory rate with respiratory compromise progressing to Acute Respiratory Distress Syndrome (ARDS).

A variety of blood-based markers have been associated with CRS including the cytokines interleukin-1 (IL-1), IL-6, IL-10, Interferon-gamma (IFN- $\gamma$ ), and Tumor Necrosis Factor-alpha (TNF- $\alpha$ ), creatinine, elevated liver enzymes, and C Reactive Protein (CRP) [1,2]. Markers such as IFN- $\gamma$ and IL-10 have good sensitivity and specificity for CRS relative to a less specific marker IL-6. However, IL-6 has been established as a central cytokine in the pathophysiology of CRS, both in animal model systems and the clinical settings [3-5]. IL-6 is a potent pro-inflammatory cytokine 
with the ability to produce cis and trans-signaling. In cis-signaling, a cell expresses the IL-6 receptor (IL-6R) on its cell surface along with the gp130 protein that contains a transmembrane domain that functions to initiate the intracellular IL-6 signaling cascade involving Janus Activated protein Kinase (JAK) and Signal Transducer and Activator of Transcription (STAT) kinases. For signaling to occur, IL-6 binds to its receptor IL-6R on a cell's surface, the complex then binds to gp130 on the same cell to initiate signaling [6]. Tran-signaling occurs when IL-6 binds to soluble IL-6R (sIL-6R), which occurs when the ADAM10 protease cleaves the membranebound IL-6R. The IL-6/sIL-6R complex can now bind to gp130 on any cell surface, independent of IL-6R expression on the target cell and induce signal transduction [6]. The prominence of IL-6 in CRS is highlighted by its' association with many of the symptoms related to the condition (i.e., fever, anemia, acute kidney injury, Natural Killer-cell dysfunction) and targeting IL-6 can ameliorate many of the symptoms accompanying CRS [5,6]. IL-6 can activate coagulation and complement cascades that are associated with Disseminated Intravascular Coagulation (DIC) and inhibit Natural Killer cells from eliminating activated $\mathrm{T}$ cells, which can lead to an uncontrolled T cell response [6]. Controlling IL-6 may be central to managing the CRS in patients.

CRS is linked to a variety of etiologies including, but not limited to, antibody-based therapies, viral infection, small molecules, effector cell therapies (i.e., Chimeric Antigen Receptor T (CAR-T cell) cell therapy), and immune checkpoint inhibitor (ICI) therapies. E-cigarette and Vaping-Associated lung disease (EVALI) and Corona Virus Disease 2019 (COVID-19) are CRS-associated diseases that have emerged recently as significant public health concerns because they can both cause life-threatening ARDS (Table 1). CRS

Table 1: Summary of CRS across various etiologies. is an immune-related adverse event (irAE) that can occur during CAR-T cell and immune checkpoint inhibitor (ICI) therapies for cancer patients. Some patients undergoing these treatments also experience adverse events that are associated with inflammation of various organs (i.e., pneumonitis, enteritis, colitis, vitiligo, etc.), and it often necessitates a pause or cessation of treatment, which could allow for disease progression. [7] Treating CRS typically involves inhibiting the immune systems, which could prevent a tumor response from immunotherapeutic agents. [8] Should the CRS in each of these illnesses be treated the same way? Since many diseases with diverse etiologies are associated with CRS, these similarities can be exploited to establish novel and effective treatment protocols for CRS. We present CRS in the context of EVALI, COVID-19, CAR-T cell therapy, and ICI therapy and discuss how each is treated, and propose a combination approach to improve the management of CRS.

\section{Discussion/Observations}

\section{CRS in Various Scenarios}

EVALI is associated with aerosolizing vitamin E acetate, typically from illicit THC-containing products, into the lung that can potentiate acute respiratory distress. The disease principally occurred in the US, affecting over 2,800 patients from all 50 states, and resulted in 68 confirmed deaths through February 18, 2020 (Table 1) [9]. After clusters of patients were identified, the source of the outbreak was quickly associated with illicit THC-containing vaping liquids, and thereafter the cases steadily declined. Patients presented with respiratory, gastrointestinal, or constitutional symptoms, with radiographic opacities indicative of one-sided or bi-lateral diffuse pneumonia, and signs of inflammation and CRS (i.e., elevated serum IL-6 and C-Reactive Protein (CRP)) [10,11].

\begin{tabular}{|c|c|c|c|c|}
\hline & CoVID-19[12] & EVALI [9] & $\begin{array}{l}\text { Effector Cell Therapies* } \\
\text { CRS-associated irAE[35] }\end{array}$ & ICI CRS-associated irAE[8] \\
\hline Cases & $\begin{array}{l}\text { Global: } 23 \text { million" } \\
\text { USA: } 5.7 \text { million" }\end{array}$ & USA: 2,807@ & $\begin{array}{c}\text { ( } \geq \text { Grade } 3 \text { ) } \\
\text { Lymphoma } \\
1-23 \% \\
\text { Leukemia: } \\
23-46 \%\end{array}$ & $58 / 80,700$ safety reports \\
\hline Deaths & $\begin{array}{l}\text { Global: } 810 \text { thousand" }{ }^{\#} \\
\text { USA: } 177 \text { thousand }{ }^{\#}\end{array}$ & USA: 68@ & $\begin{array}{l}\text { Lymphoma } 0-1.8 \% \\
\text { Leukemia } 0-1.9 \%\end{array}$ & $2(3.4 \%)$ \\
\hline $\begin{array}{l}\text { Associated Causative } \\
\text { Agent(s) }\end{array}$ & SARS-CoV-2 & VEA & $\begin{array}{l}\text { CAR-T cells } \\
\text { BiTE }\end{array}$ & $\begin{array}{l}\text { PD1 antagonist } \\
\text { PD-L1 antagonist } \\
\text { CTLA-4 antagonist }\end{array}$ \\
\hline Risk Factors & Comorbidities & $\begin{array}{c}\text { Age } \\
\text { Comorbidities }\end{array}$ & $\begin{array}{c}\text { Comorbidities } \\
\text { Large tumor burden } \\
\text { Early-onset CRS }(<3 \text { days })\end{array}$ & \\
\hline
\end{tabular}

*Effector cell therapies include CAR-T and BiTE cell treatments; \#: Numbers through 8/24/2020; @: Number through 2/18/2020; irAE: immune-related Adverse Event; ICl: Immune Checkpoint Inhibitor; SARS-CoV-2: Severe Acute Respiratory Syndrome CoronaVirus-2; VEA: Vitamin E Acetate. 
Table 2: ASTCT Grading Scale for Cytokine Release Syndrome (CRS) [36].

\begin{tabular}{|c|c|}
\hline Grade & Characteristics \\
\hline 1 & $\begin{array}{l}\qquad \text { Fever } \geq 38^{\circ} \mathrm{C} \\
\text { Could include constitutional symptoms, i.e., myalgia, malaise, etc. }\end{array}$ \\
\hline 2 & $\begin{array}{l}\qquad \text { Fever } \geq 38^{\circ} \mathrm{C} \\
\text { Hypotension using not requiring vasopressors } \\
\text { Hypoxia that may necessitate some oxygen supplementation, i.e., low-flow nasal cannula. }\end{array}$ \\
\hline 3 & $\begin{array}{l}\qquad \text { Fever } \geq 38^{\circ} \mathrm{C} \\
\text { Hypotension requiring the use of a vasopressor (excluding vasopressin) } \\
\text { Hypoxia that requires oxygen supplementation, i.e., high-flow nasal cannula. }\end{array}$ \\
\hline 4 & $\begin{array}{l}\qquad \text { Fever } \geq 38^{\circ} \mathrm{C} \\
\text { Hypotension requiring multiple vasopressors (including vasopressin) } \\
\text { Hypoxia that requires positive pressure or intubation. }\end{array}$ \\
\hline 5 & Death \\
\hline
\end{tabular}

ASTCT: American Society for Transplantation and Cellular Therapy.

Table 3A: Major therapies available for CRS conditions.

\begin{tabular}{|c|c|c|c|}
\hline COVID-19 & EVALI & Effector Cell Therapies irAE & ICI irAE \\
\hline Corticosteroids & Corticosteroids & Corticosteroids & Corticosteroids \\
Convalescent Plasma & & IL-6 and IL-6R antagonists & \\
Remdesivir & & & \\
\hline
\end{tabular}

COVID-19: Coronavirus Disease 2019; EVALI: E-cigarette and Vaping Associated Lung Injury; irAE: immune-related Adverse Events; ICI: Immune Checkpoint Inhibitors.

Table 3B: Concept for a clinical trial.

\section{Proposed Therapy/Clinical Trial}

Combination of IL-6 or IL-6R antagonist and low dose corticosteroids for management of CRS

COVID-19 results from an infection with the Severe Acute Respiratory Syndrome-2 Corona Virus (SARS-CoV-2) virus in the lungs. There have been over 4.7 million COVID-19 cases with 156,000 deaths in the US, and over 18 million cases with 700,000 deaths worldwide, through August 3, 2020 (Table 1) [12]. Patients with severe cases of COVID-19 display a hyperactive immune response indicative of a cytokine storm. A variety of inflammatory markers (i.e., C-Reactive Protein, Lactate Dehydrogenase) and cytokines (i.e., IL-6, IL-1) are elevated in the plasma of these patients [13].

The major trend in oncology is augmenting the immune system to generate a robust anti-tumor response. For solid tumors, this can be done by using ICI targeting the CTLA-4 or PD-1/PD-L1 pathways. These agents, i.e., Nivolumab, can produce immune-related adverse events caused by CRS [7]. Although CRS in patients treated with ICI is rare, the large number of patients who are being prescribed these drugs makes these events a significant problem for the modern oncologist. Patients tend to present with fever, tachypnea, tachycardia, hypotension, and organ failure within 24-48 hours or weeks following ICI treatment. CRS symptoms are commonly associated with elevated blood CRP, creatinine, and IL-6 [6,14].

Oncologists can also manipulate the immune system of cancer patients by adaptive $\mathrm{T}$ cell-based therapies, i.e., CAR-T cell and Bi-specific $\mathrm{T}$ cell Engager (BiTE) cell therapy. Following these therapies, it is estimated that $50 \%$ of patients can develop grade 3-4 CRS symptoms (Table 2) as a result of excessive cytokine production, especially IL-1, and IL-6, causing pyrexia, hypotension, pulmonary edema, reduced renal function, and cardiovascular toxicities [3,15]. CRS symptoms typically occur between 6-20 days following cell infusion, and coincident with a greater than ten-fold increases in IFN- $\gamma$ and IL- 6 and a greater than four-fold increase in TNF- $\alpha$ [6]. The symptoms can progress leading to ARDS and MOF if the condition is not managed. 


\section{Management of CRS}

CRS is the result of an over-activation of the immune system therefore, the condition is commonly treated with corticosteroids. Therapeutics used to manage CRS from different scenarios are summarized in Table 3A. The steroids potently suppress the expression of many cytokines effectively slowing down the immune response. Muting the immune system in patients where CRS is driving the disease symptoms, such as EVALI and COVID-19, with steroids can be beneficial. Corticosteroids have been used to treat patients with severe cases of EVALI, but the efficacy of the steroids for treating EVALI patients has not been determined in randomized clinical trials [16-18]. Steroids also seem to be beneficial for patients with COVID-19 with CRS. The corticosteroid dexamethasone was the first drug targeting the cytokine storm to show an improvement in survival for COVID-19 patients with severe disease and especially those on respiratory support [19].

Corticosteroids, however, can impede a successful antitumor response of checkpoint inhibitors and effector $\mathrm{T}$ cell therapies, hindering the treatment's efficacy. This is especially the case when steroids are given early in the treatment course or if patients are on steroids for unrelated autoimmune conditions before treatment [20]. The use of corticosteroids can reduce progression-free survival and overall survival in non-small cell lung cancer (NSCLC) patients treated with drugs targeting the PD-1 and PD-L1 pathways [20]. Severe irAE (stage 3 and 4; Table 2) in patients receiving ICI must be managed effectively, and currently, the primary strategy is to use high-dose corticosteroids with possible detriment to antitumor efficacy. Fortunately, the recurrence rate of irAE in cancer patients may be low, so it seems that it may be possible to restart checkpoint inhibitors in patients who initially had irAE [21].

CRS associated with CAR-T cell or BiTE therapies can be mitigated by low doses of corticosteroids (i.e., dexamethasone), while still maintaining the anti-tumor response (Table 3A) [22]. Therefore, these drugs can effectively improve the quality of life for patients receiving CAR-T cell therapies, but the current data does not show these drugs improve the outcome of the CAR-T cell treatment in patients [22]. The management of CRS in situations where the stimulus is a pathogen or other external agent (i.e., vitamin E acetate, VEA), corticosteroids seem to be effective, but for cancer patients undergoing ICI or effector T cell therapies, broad inhibition of the immune system is sub-optimal as they can alter the anti-tumor effect of these agents. A targeted approach that controls CRS while allowing an appropriate immune response would be an effective tool to manage the condition caused by exogenous agents and immunotherapeutic agents because corticosteroids, especially high doses, can lead to an increased risk for infection [23]

\section{Targeting the IL-6 pathway}

The IL-6 pathway is an ideal target candidate to manage the effects of CRS. Many of the symptoms associated with CRS are related to IL- 6 and modulating IL- 6 or the IL- 6 pathway can moderate or abrogate the symptoms of CRS. A variety of modalities have been established to target the IL- 6 pathway including antiIL-6R antibodies (tocilizumab), anti-IL-6 antibodies (siltuximab), inhibition of trans-IL-6 signaling with Sgp130-Fc, and JAK/STAT inhibition (ruxolitinib) [6]. A variety of clinical studies have been established to study the efficacy of IL- 6 and IL-6R antagonists in COVID-19 patients, however, data from these trials are not yet mature. Small, retrospective studies suggest that tocilizumab may have some efficacy in managing CRS in COVID-19 patients [24-26]. Tocilizumab is effective and has received FDA approval for treating CRS associated with CAR-T cell therapy in adults and children over 2 years of age [27]. There is little data available on the application of tocilizumab to control ICI-induced CRS. In a small study, tocilizumab in combination with steroids versus steroids alone showed that the combination could effectively manage CRS symptoms associated with nivolumab treatment, but there was no change in median survival between the groups [28]. Tocilizumab may be effective at alleviating ICI-associated joint inflammation [29,30]. In contrast to tocilizumab, siltuximab has not been studied in the context of firstline treatment for CAR-T cell or ICI-induced CRS, but it has been applied as a third-line treatment for CAR-T cell-associated CRS following the failure of tocilizumab and steroids [31]. Siltuximab has shown some promise in treating severe COVID-19 induced CRS, but these results are preliminary and require confirmation in prospective clinical trials [32].

Targeting the IL-6 down-stream effector proteins JAK $1 / 2$ with the small molecule inhibitor ruxolitinib has also shown efficacy in inhibiting the effects of CRS. A small cohort study of COVID-19 patients showed the ruxolitinib was able to reduce inflammation in 12 or 14 patients [33]. Further clinical studies are ongoing to study the drug's efficacy in COVID-19 patients. While ruxolitinib has not been tested on CRS associated with ICI, it has shown promise in treating CRS following CAR-T cell therapy. In a mouse model, ruxolitinib was able to inhibit CRS while producing a negligible effect on the anti-tumor response of the CAR-T cell cells [34-36]. Clinical studies are needed to determine the efficacy of ruxolitinib to treat CAR-T cell-associated CRS in humans.

\section{A combination-based approach to manage CRS in cancer}

Treating patients with CRS can be a difficult proposition as the etiology is multifactorial. The cause and desired outcome play an important role in how it should be managed. For exogenous agents like SARS-CoV-2 or VEA, a broad-based approach with 
corticosteroids seems to be effective. However, with immunemodulatory treatments in cancer where a robust anti-tumor immune response is required, ICI and adaptive $\mathrm{T}$ cell therapies, a targeted approach may be more beneficial. Targeting the IL- 6 pathway has shown promise, as these tend to allow the immune cells to continue to function with anti-tumor effects [31,32]. The success of targeting the IL- 6 pathway with tocilizumab to control CAR-T cell-associated CRS highlights that IL- 6 is one of the key factors responsible for the symptoms associated with the syndrome. More clinical data are needed to better understand CRS and how it can be controlled, especially in the context of IL- 6 production and the cause of inflammation. We suggest that combining lower doses of corticosteroids with IL-6 targeting agents may prove to be more effective in controlling CRS than each agent individually in cancer patients receiving immunotherapies (Table 3B). By limiting the broad-spectrum immune inhibition of steroids and supplementing it with an IL-6 targeting agent, a synergistic effect may occur where CRS symptoms are managed, and immune cell anti-tumor responses are preserved.

\section{Conclusion}

The clinical management of CRS from patients with all the etiologies discussed herein may be optimized by combining low dose corticosteroids with agents targeting IL-6 or the IL-6 receptor. There is currently a trial underway to compare tocilizumab or siltuximab with or without corticosteroids in patients with COVID-19 (NCT04486521). This study will require close monitoring so that it could inform and inspire new and more effective treatment options for CRS in general.

\section{Acknowledgement}

None.

\section{Conflict of Interest}

No conflict of interest.

\section{References}

1. Shimabukuro Vornhagen A, Gödel P, Subklewe M, Hans Joachim Stemmler, Max Schlaak, et al. (2018) Cytokine release syndrome. J Immunother Cancer 6(1): 56.

2. Xu XJ, Tang YM (2014) Cytokine release syndrome in cancer immunotherapy with chimeric antigen receptor engineered T cells. Cancer Lett 343(2): 172-178

3. Norelli M, Camisa B, Barbiera G, Laura Falcone, Ayurzana Purevdorj, et al. (2018) Monocyte-derived IL-1 and IL-6 are differentially required for cytokine-release syndrome and neurotoxicity due to CAR T cells. Nat Med 24(6): 739-748.

4. Theodoros Giavridis, van der Stegen SJC, Eyquem J, Hamieh M, Piersigilli A, et al. (2018) CAR T cell-induced cytokine release syndrome is mediated by macrophages and abated by IL-1 blockade. Nat Med 24(6): 731738.

5. Shimizu M (2019) Clinical Features of Cytokine Storm Syndrome. Cytokine Storm Syndrome. Springer International Publishing pp: 31-41.
6. Barrett D (2019) IL-6 Blockade in Cytokine Storm Syndromes. Cytokine Storm Syndrome. Springer International Publishing pp: 561-568.

7. Postow MA, Sidlow R, Hellmann MD (2018) Immune-Related Adverse Events Associated with Immune Checkpoint Blockade. N Engl J Med 378(2): 158-168

8. Ceschi A, Noseda R, Palin K, Verhamme K (2020) Immune Checkpoint Inhibitor-Related Cytokine Release Syndrome: Analysis of WHO Global Pharmacovigilance Database. Front Pharmacol 11: 557.

9. (2020) Prevention CfDCa. Outbreak of Lung Injury Associated with the Use of E-Cigarette, or Vaping, Products.

10. Siegel DA, Jatlaoui TC, Koumans EH, Emily A Kiernan, Mark Layer, et al. (2019) Update: Interim Guidance for Health Care Providers Evaluating and Caring for Patients with Suspected E-cigarette, or Vaping, Product Use Associated Lung Injury - United States, October 2019. MMWR Morb Mortal Wkly Rep 68(41): 919-927.

11. Rice SJ, Hyland V, Behera M, Ramalingam SS, Bunn P, et al. (2020) Guidance on the clinical management of E-cigarette or Vaping Associated Lung Injury. J Thorac Oncol 15(11): 1727-1737.

12. University JH. COVID-19 Dashboard by the Center for Systems Science and Engineering (CSSE) at Johns Hopkins University (JHU), Maryland, USA.

13. Mehta P, McAuley DF, Brown M, Emilie Sanchez, Rachel S Tattersall, et al. (2020) COVID-19: consider cytokine storm syndromes and immunosuppression. Lancet 395(10229): 1033-1034.

14. Sharma N, Stroud CRG, Walker PR, Cynthia R Cherry, Sulochana, et al. (2016) Systemic inflammatory response syndrome (SIRS) with immune checkpoint inhibitors. Journal of Clinical Oncology 34(15 suppl): 30613061.

15. Fitzgerald JC, Weiss SL, Maude SL, David M Barrett, Simon F Lacey, et al. (2017) Cytokine Release Syndrome After Chimeric Antigen Receptor T Cell Therapy for Acute Lymphoblastic Leukemia. Crit Care Med 45(2): e124-e131.

16. Blagev DP, Harris D, Dunn AC, Guidry DW, Grissom CK, et al. (2019) Clinical presentation, treatment, and short-term outcomes of lung injury associated with e-cigarettes or vaping: a prospective observational cohort study. Lancet 394(10214): 2073-2083.

17. Layden JE, Ghinai I, Pray I, Anne Kimball, Mark Layer, et al. (2020) Pulmonary Illness Related to E-Cigarette Use in Illinois and Wisconsin-Final Report. N Engl J Med 382(10): 903-916.

18. Evans ME, Twentyman E, Click ES, Alyson B Goodman, David N Weissman, et al. (2020) Update: Interim Guidance for Health Care Professionals Evaluating and Caring for Patients with Suspected E-cigarette, or Vaping, Product Use-Associated Lung Injury and for Reducing the Risk for Rehospitalization and Death Following Hospital Discharge-United States, December 2019. MMWR Morb Mortal Wkly Rep 68(5152): 11891194.

19. Kupferschmidt K (2020) A cheap steroid is the first drug shown to reduce death in COVID-19 patients. AAAS.

20. Della Corte CM, Morgillo F (2019) Early use of steroids affects immune cells and impairs immunotherapy efficacy. ESMO Open 4(1): e000477.

21. Dolladille C, Ederhy S, Sassier M, Jennifer Cautela, Franck Thuny, et al. (2020) Immune Checkpoint Inhibitor Rechallenge After Immune-Related Adverse Events in Patients with Cancer. JAMA Oncol 6(6): 865-871.

22. Chen H, Wang F, Zhang P, Yilin Zhang, Yinxia Chen, et al. (2019) Management of cytokine release syndrome related to CAR-T cell therapy. Front Med 13(5): 610-617.

23. Ozen S, Esenboga S (2019) Alternative Therapies for Cytokine Storm Syndromes. Cytokine Storm Syndrome. Springer International Publishing pp: 581-593. 
24. Andrianopoulos I, Papathanasiou A, Papathanakos G, Chaidos A, Koulouras V. (2020) Tocilizumab's efficacy in patients with Coronavirus Disease 2019 (COVID-19) is determined by the presence of cytokine storm. Journal of Medical Virology.

25. Guaraldi G, Meschiari M, Cozzi-Lepri A, Jovana Milic, Roberto Tonelli, et al. Tocilizumab in patients with severe COVID-19: a retrospective cohort study. Lancet Rheumatol 2(8): e474-e484.

26. Xu X, Han M, Li T, Wei Sun, Dongsheng Wang, et al. (2020) Effective treatment of severe COVID-19 patients with tocilizumab. Proc Natl Acad Sci U S A 117(20): 10970.

27. Le RQ, Li L, Yuan W, Stacy S Shord, Lei Nie, et al. (2018) FDA Approval Summary: Tocilizumab for Treatment of Chimeric Antigen Receptor T Cell-Induced Severe or Life-Threatening Cytokine Release Syndrome. Oncologist 23(8): 943-947.

28. Stroud CRG, Hegde A, Naqash AR, et al. (2017) OA03.05 Tocilizumab for the Management of Immune Mediated Adverse Events Secondary to PD-1 Blockade: Overall Survival Analysis: Topic: Medical Oncology. Journal of Thoracic Oncology 12(11): S1554-S1555.

29. Zhou J, Wang H, Guo X, Qian Wang, Lian Duan, et al. (2020) Management of immune checkpoint inhibitor-related rheumatic adverse events. Thoracic Cancer 11(1): 198-202.

30. Kim ST, Tayar J, Trinh VA, Maria Suarez Almazor, Salvador Garcia, et al. (2017) Successful treatment of arthritis induced by checkpoint inhibitors with tocilizumab: a case series. Ann Rheum Dis 76(12): 2061-2064.
31. Teachey DT, Bishop MR, Maloney DG, Grupp SA (2018) Toxicity management after chimeric antigen receptor T cell therapy: one size does not fit 'ALL'. Nat Rev Clin Oncol 15(4): 218

32. Gritti G, Raimondi F, Ripamonti D, Ivano Riva, Francesco Landi, et al (2020) IL-6 signalling pathway inactivation with siltuximab in patients with COVID-19 respiratory failure: an observational cohort study. medRxiv 20048561

33. La Rosée F, Bremer HC, Gehrke I, et al. (2020) The Janus kinase 1/2 inhibitor ruxolitinib in COVID-19 with severe systemic hyperinflammation. Leukemia 34(7): 1805-1815.

34. Kenderian SS, Ruella M, Shestova O, Miriam Y Kim, Michael Klichinsky, et al. (2016) Ruxolitinib Prevents Cytokine Release Syndrome after CART Cell Therapy without Impairing the Anti-Tumor Effect in a Xenograft Model. Blood 128(22): 652-652.

35. Santomasso B, Bachier C, Westin J, Rezvani K, Shpall EJ (2019) The Other Side of CAR T-Cell Therapy: Cytokine Release Syndrome, Neurologic Toxicity, and Financial Burden. Am Soc Clin Oncol Educ Book 39: 433-444.

36. Lee DW, Santomasso BD, Locke FL, Armin Ghobadi, Cameron J Turtle, et al. (2019) ASTCT Consensus Grading for Cytokine Release Syndrome and Neurologic Toxicity Associated with Immune Effector Cells. Biol Blood Marrow Transplant 25(4): 625-638. 